\title{
INSUFICIÊNCIA ADRENAL CRÔNICA E AGUDA
}

\author{
ADRENALINSUFFICIENCY
}

Margaret de Castro ${ }^{1}$ \& Lucila L. K. Elias ${ }^{2}$

\begin{abstract}
'Docente. ${ }^{2}$ Médica Assistente. Divisão de Endocrinologia e Metabologia. Departamento de Clinica Médica. Faculdade de Medicina de Ribeirão Preto - USP.

CorResPondÊnCIA: Margaret de Castro. Divisão de Endocrinologia e Metabologia Departamento de Clínica Médica da FMRP-USP. Av Bandeirantes, 3900 CEP 14049-900 Ribeirão Preto SP. fax: 16-6331144 - fone: 16-6022654 - email: castrom@fmrp.usp.br
\end{abstract}

CASTRO M \& ELIAS LLK. Insuficiência adrenal crônica e aguda. Medicina, Ribeirão Preto, 36: 375-379, abr./ dez. 2003.

RESUMO - Os glicocorticóides apresentam um importante papel na regulação metabólica, nos sistemas cardiovascular, imune, nervoso, e na resposta adaptativa ao estresse. A insuficiência adrenal pode ser causada por uma doença primária da adrenal (baixas concentrações plasmáticas de cortisol e altas de ACTH) ou secundária a doenças do eixo hipotálamoipofisário (baixas concentrações plasmáticas de cortisol e de ACTH). A falta crônica de glicocorticóide leva a sintomas insidiosos e inespecíficos (mal-estar geral, fraqueza, inapetência, perda de peso, queixas gastrintestinais). Adicionalmente, quando há também deficiência de mineralocorticóide, a hipotensão, síncope, desidratação e choque cardiocirculatório, com hiponatremia e hipercalemia podem associar-se ao quadro clínico. O tratamento consiste na reposição de cortisol (hidrocortisona VO, 12 a $15 \mathrm{mg} / \mathrm{m}^{2}$ de superfície corporal, acetato de cortisona VO, 25mg/dia e prednisona VO, 5 a $10 \mathrm{mg} / \mathrm{dia}$ ) na insuficiência adrenal secundária e de cortisol e minelocorticóide ( $9 \alpha$ fluorohidrocortisona VO, $0,1 \mathrm{mg} / \mathrm{dia}$ ) na primária. Doenças infecciosas, traumas e cirurgias podem precipitar uma crise aguda, chamada crise addisoniana, situação esta de risco de vida, quando não tratada. $O$ tratamento consiste de reposição do volume intravascular com cloreto de sódio (soro fisiológico 0,9\% 2 L/hora) e glicocorticóides (100 mg EV de hidrocortisona a cada 6 h). Desde que a crise addisoniana é freqüentemente desencadeada por processo infeccioso, o diagnóstico de infecção deve ser confirmado e, se presente, o uso de antibioticoterapia deve ser preconizado.

UNITERMOS - Insuficiência Adrenal. Cortisol. ACTH

\section{1- INTRODUÇÃO}

O córtex adrenal produz glicocorticóides, mineralocorticóides e andrógenos, e a medula produz catecolaminas. Os glicocorticóides regulam o metabolismo dos carboidratos agindo como contra-reguladores da insulina, estimulando a gliconeogênese hepática e a glicogenólise. Diminuem, ainda, a utilização periférica de glicose, atuando sobre o receptor da in- sulina e diminuindo os transportadores de glicose. No metabolismo dos lipídeos, quando administrados agudamente, aumentam a lipólise, porém a exposição crônica resulta no acúmulo de gorduras nas regiões supraclaviculares, dorsocervical e tronco. Estimulam a mobilização de proteínas musculares, inibindo a síntese protéica e acelerando a proteólise, resultando em importante miopatia. Atuam ainda nos ossos, no crescimento esquelético, no sistema nervoso central, no 
comportamento, e como antiinflamatórios e imunossupressores, modulando a migração de células imunocompetentes e a liberação de citocinas. Além dos efeitos metabólicos dos glicocorticóides, há os efeitos de contra-regulação negativa dos mesmos sobre o eixo hipotalamoipófiseadrenal (HHA), em que a redução do glicocorticóide circulante resulta num aumento compensatório do ACTH hipofisário e plasmático. Os glicocorticóides apresentam, ainda, um importante papel na resposta adaptativa ao estresse.

A síntese de aldosterona ocorre exclusivamente na zona glomerulosa e suas principais ações estão relacionadas com a manutenção da homeostase hidroeletrolítica, atuando nos túbulos contornados distais e ductos coletores, estimulando a reabsorção de sódio e secreção de potássio e hidrogênio. Os andrógenos adrenais são pouco potentes e não efetivos, até serem convertidos em testosterona e $5 \alpha$-diidrotestosterona, em tecidos periféricos.

\section{2- INSUFICIÊNCIA ADRENAL CRÔNICA}

A insuficiência adrenal pode ser dividida em 2 tipos: a insuficiência adrenal primária (Doença de Addison) é causada por uma doença primária da adrenal e é caracterizada por baixa produção de cortisol e altas concentrações de ACTH. Por outro lado, a insuficiência adrenal secundária é causada por doenças que comprometem o eixo hipotalamoipofisário e é caracterizada por baixa produção de cortisol e de ACTH. A falência da produção de cortisol e aldosterona é observada na insuficiência adrenal primária, enquanto, na secundária, a deficiência é apenas de cortisol, pois com as adrenais preservadas, há a manutenção da secreção de aldosterona que é regulada primariamente pelo sistema reninangiotensina.

As diferentes causas de insuficiência adrenal primária podem ser agrupadas em alterações do desenvolvimento ou destruição da glândula e deficiência na síntese de cortisol (Tabela I) e podem ser de causa genética ou adquirida. Na criança, a causa mais comum é a hiperplasia adrenal congênita, doença autossômica, recessiva, decorrente da deficiência de uma das cinco enzimas envolvidas na síntese de cortisol.
Na vida adulta, as causas mais comuns de insuficiência adrenal primária, incluem a adrenalite auto-imune e, em nosso meio, a tuberculose e a paracoccidioidomicose. As causas secundárias de insuficiência adrenal estão apresentadas na Tabela II. A principal causa é a supressão do eixo HHA, que ocorre como resultado da administração exógena de glicocorticóides. Os sintomas, geralmente, iniciam após $48 \mathrm{~h}$ da suspensão da medicação.

A falta crônica de glicocorticóide leva a sintomas inespecíficos, tais como mal-estar geral, fraqueza, inapetência, perda de peso, queixas gastrintestinais, como náusea, vômitos, dor abdominal e diarréia alternada ou não com constipação intestinal. São sintomas comuns em muitas outras doenças, entretanto, a insuficiência adrenal deve ser sempre considerada no diagnóstico diferencial. A hipoglicemia pode ser uma manifestação inicial da insuficiência adrenal. Adicionalmente, quando há também deficiência de mineralocorticóides, freqüentemente observada na insuficiência adrenal primária, a hipotensão, síncope, desidratação e o choque cardiocirculatório, com hiponatremia e hipercalemia, podem associar-se ao quadro
Tabela I: Causas de insuficiência adrenal primária

Idiopática (incluindo insuficiência poliglandular auto-imune) $65 \%$

Tuberculose

Outras Causas

Infecção por fungos (Ex: paracoccidioido micose)

Hemorragia adrenal

Metástases

Sarcoidose

Amiloidose

Adrenoleucodistrofia

Adrenomieloneuropatia

SIDA

Hiperplasia adrenal congênita

Hipoplasia adrenal congênita

Deficiência familiar de glicocorticóide

Uso de medicação (Ex. mitotane, aminoglutetimida) 
Tabela II: Causas de insuficiência adrenal secundária

1. Supressão do eixo hipotalamoipófiseadrenal

A. Exógena

- Glicocorticóide

- ACTH

B. Endógena: Pós-operatório de síndrome de Cushing curada

2. Lesões hipotalâmicas ou hipofisárias

A. Neoplasias

- Tumor hipofisário

- Tumor metastático

B. Craniofaringeoma

C. Necrose isquêmica

D. Hipofisite auto-imune

E. Infecção

- Tuberculose

- Nocardia

- Actinomicose

3. Sarcoidose

4. Trauma cranioencefálico

5. Deficiência isolada de $\mathrm{ACTH}$ associada a sinais e sintomas decorrentes da deficiência de outros eixos hipofisários.

As anormalidades laboratoriais incluem anemia normocítica e normocrômica, linfocitose relativa com eosinofilia e discreta acidose metabólica. Anormalidades eletrolíticas incluem hiponatremia e hipercalemia na insuficiência adrenal primária e somente hiponatremia na secundária. Concentrações de cortisol plasmático $(<5 \mu \mathrm{g} / \mathrm{dl})$ ou nas suas formas livres (urinário ou salivar) baixas e concentrações elevadas de ACTH (> 100 pg/ml) confirmam insuficiência adrenal primária. O teste da hipoglicemia insulínica (ITT) consiste na indução de hipoglicemia $(<40 \mathrm{mg} / \mathrm{dl})$ pela aplicação de $1 \mathrm{U} / \mathrm{Kg}$ de peso de insulina regular por via endovenosa. É um potente estímulo para liberação de cortisol, entretanto, apresenta algumas contra-indicações, como a presença de crise convulsiva, coronariopatia em idosos. É um teste utilizado principalmente para avaliar a integridade do eixo hipotalamoipófiseadrenal. Um teste anormal indica lesão em qualquer parte do eixo que, quando associado às concentrações plasmáticas

clínico. Na mulher, a insuficiência adrenal resulta em perda dos pelos pubianos e axilares. Pacientes com insuficiência adrenal grave ou de longa duração podem apresentar mialgias e artralgias difusas, além de sintomas psiquiátricos, como perda de memória, delírio, depressão e psicose. Todos estes sinais e sintomas não permitem a diferenciação entre a causa primária e secundária da insuficiência adrenal, no entanto, o achado objetivo de hiperpigmentação, devido ao excesso de ACTH, obtido pela semiologia da pele, mucosas e cicatrizes, indica a causa primária da doença. Hiperpigmentação cutânea está presente em cerca de $90 \%$ dos casos de insuficiência adrenal primária. A presença de vitiligo, candidíase mucocutânea sugerem a etiologia auto-imune da doença. Ainda, a insuficiência adrenal primária, de causa autoimune pode estar associada à tireoidite de Hashimoto, hipoparatireoidismo, diabetes mellitus do tipo 1 e falência ovariana primária, constituindo a síndrome poliglandular auto-imune. Cabe salientar que a deficiência de CRH/ACTH isolada é rara, portanto, a insuficiência adrenal de causa secundária, está, geralmente, de ACTH baixas, diagnostica insuficiência adrenal dovenosa de 250 ug de ACTH exógeno -Synacten ${ }^{\mathrm{R}}$, Ciba) tem se tornado o principal teste para diagnóstico de insuficiência adrenal primária. Resposta do cortisol acima de 19ug/dl aos testes de ITT e ACTH exógeno indica reserva adrenal preservada. A realização de tais testes não é factível na sala de urgência, portanto, na suspeita de crise addisoniana, a Divisão de Endocrinologia recomenda a coleta de sangue para determinação imediata de sódio, potássio, e estoque do plasma para posterior dosagem de ACTH e cortisol, seguida da administração de glicocorticóide, em casos de risco de vida.

O tratamento da insuficiência adrenal crônica consiste na reposição de cortisol na insuficiência adrenal secundária e cortisol e mineralocorticóide na primária. Muitas preparações de glicocorticóides são disponíveis para esse fim. Nos EUA, a droga de escolha é a hidrocortisona oral (12 a $15 \mathrm{mg} / \mathrm{m}^{2}$ de superfície corporal). No Brasil, existe uma boa experiência com o uso de acetato de cortisona ( $25 \mathrm{mg} /$ dia) e mesmo secundária. O teste do ACTH exógeno (injeção en- 
com prednisona (5 a $10 \mathrm{mg} / \mathrm{dia}$ ). Cabe ressaltar que os esteróides sintéticos, como a prednisona ou dexametasona, não apresentam efeito mineracorticóide e, portanto seriam mais apropriadas para intervenções farmacológicas nas doenças inflamatórias que para o uso em reposição fisiológica. A única preparação mineralocorticóide disponível é a $9 \alpha$ fluoroidrocortisona (nome comercial nos EUA e Europa Florinef $^{\mathrm{R}}$ - Bristol-Myers Squibb, não disponível comercialmente Brasil, necessitando de formulação em farmácia de manipulação), que geralmente, é usada na dose de $0,1 \mathrm{mg} /$ dia.

A monitorização terapêutica dever ser realizada pela avaliação clínica. Sub-tratamento é evidenciado pela presença ou manutenção dos sintomas. Por outro lado, o tratamento excessivo resulta nas manifestações clínicas de hipercortisolismo, como ganho de peso, estrias, fraqueza, hipertensão arterial e, na criança, desaceleração da velocidade de crescimento. A adequação do tratamento mineralocorticóide pode ser avaliada pela monitorização da pressão arterial, concentrações séricas de sódio e potássio e atividade de renina plasmática.

\section{3- INSUFICIÊNCIA ADRENAL AGUDA}

As manifestações da insuficiência adrenal são geralmente insidiosas, entretanto, a presença de fatores de estresse, como doenças infecciosas, traumas e cirurgias, podem precipitar uma crise aguda, chamada crise addisoniana. A insuficiência adrenal aguda pode resultar em uma situação de risco de vida, quando não tratada.Assim, o reconhecimento, diagnóstico e tratamento correto e precoce é de fundamental importância na prática clínica. Para minimizarmos os riscos de crise adrenal ou demora na introdução de medidas adequadas, todo paciente deve possuir identificação de portador de insuficiência adrenal e de orientação das medidas necessárias em situações de risco. Adicionalmente, em vigência de infecções, sem repercussões sistêmicas importantes, o paciente deve ser orientado a aumentar a dose habitual do glicocorticóide em duas ou três vezes, enquanto persistir o quadro de infecção. Em casos de cirurgias eletivas, recomenda-se a utilização de hidrocortisona endovenosa na dose de $100 \mathrm{mg}$ na indução anestésica, seguida de 50 a $100 \mathrm{mg}$ de $6 / 6 \mathrm{~h}$, com redução progressiva a partir do segundo dia, de acordo com a recuperação do paciente.
A insuficiência adrenal aguda deve ser considerada em qualquer paciente que esteja em uso de glicocorticóides, ou tenha uma doença sistêmica que pode se associar com insuficiência adrenal, como câncer metastático, SIDA, tuberculose, na presença de febre inexplicada, dor abdominal, e hipotensão ortostática. Em uma situação extrema, o paciente pode apresentar choque cardiocirculatório, não responsivo à reposição de volumes e drogas vasoativas, taquicardia supraventricular, redução do débito cardíaco e diminuída resistência periférica. Nessas condições, o sangue do paciente deve ser coletado para dosagens de eletrólitos, hemograma, cortisol e, se possível, de ACTH. Caso haja hiponatremia e hipercalemia, o paciente deve ser tratado como portador de insuficiência adrenal, pois é imprudente esperar pela confirmação do diagnóstico.

O tratamento consiste de reposição do volume intravascular, cloreto de sódio e glicocorticóides. A infusão de salina deve ser reposta, tão rápido quanto o estado cardiovascular permita. Geralmente, inicia-se com a infusão de soro fisiológico $0,9 \% 2 \mathrm{~L}$ /hora, nas primeiras horas do tratamento. Após a melhora da hipotensão, a taxa de infusão pode retornar a 3 a 41 de salina isotônica por dia. O uso de soro glicofisiológico pode ser útil, pois tais pacientes, geralmente, vêm de períodos longos de anorexia e, também, pela deficiência de glicocorticóides, apresentam tendência a hipoglicemia.

Glicocorticóides (100 mg de hidrocortisona cada $6 \mathrm{~h})$ devem ser administrados pela via intravenosa. Se o diagnóstico de insuficiência adrenal for confirmado, haverá uma dramática melhora em $12 \mathrm{~h}$ após o tratamento, e a dose de hidrocortisona poderá ser reduzida progressivamente, retornando à dose de manutenção em 2 a 3 dias. Caso o diagnóstico não se confirme, o regime terapêutico deve ser descontinuado.

O uso de antibioticoterapia, na fase inicial do tratamento da insuficiência adrenal aguda é controvertido. A crise addisoniana é freqüentemente desencadeada por um processo infeccioso e, alguns autores tratam os pacientes, desde o início, com antibióticos. Entretanto, é também freqüente o paciente apresentar febre durante a crise de insuficiência adrenal aguda, independente de presença de infecção. Portanto, há serviços, incluindo o nosso, que esperam as primeiras horas de tratamento e analisam a evolução clínica dos pacientes antes da introdução de antibióticos. 
CASTRO M \& ELIAS LLK. Adrenal insufficiency. Medicina, Ribeirão Preto, 36: 375-379, apr./dec. 2003.

ABSTRACT- Glucocorticoids have an important role in the metabolic regulation, in the cardiovascular, immune, and nervous systems, and in the adaptive response to stress. The adrenal insufficiency can be caused by a primary disease of the adrenal (low concentration of cortisol and high ACTH levels) or secondary to the hypothalamic-pituitary dysfunction (low cortisol and ACTH levels). Chronic glucocorticoid deficiency leads to insidious and unspecific symptoms (weakness, fadigue, gastrintestinal symptoms, anorexia, loss of weight). In the presence of mineralocorticoid deficiency clinical findings are also associated to hypotension, syncope, dehydration and circulatory shock, with hiponatremia and hiperkalemia. Management consists of cortisol replacement (hydrocortisone PO, $12-15 \mathrm{mg} / \mathrm{m}^{2}$, cortisone acetate PO, 25mg/day or prednisone PO, 5-10 mg/ day) for secondary adrenal insufficiency and cortisol and mineralocorticoid replacement $(9 \alpha$ fluorohydrocortisone PO, $0.1 \mathrm{mg} / \mathrm{dia}$ ) for primary cause of adrenal insufficiency. Infections, traumas and surgeries can precipitate an acute crisis, Addisonian crisis, a life- threatening situation if not treated. Treatment consists of replacing intravascular volume with sodium chloride $(0.9 \%$ $\mathrm{NaCl} 2 \mathrm{~L} / \mathrm{h}$ ) and glucocorticoid (100 mg IV of hydrocortisone every 6 hours). Addisonian crisis is frequently associated with infection, therefore this diagnosis must be confirmed and properly treated with antibiotics.

UNITERMS - Adrenal Insufficiency. Cortisol. ACTH

\section{BIBLIOGRAFIA RECOMENDADA}

1 - TEM S; NEW M \& MACLAREN N. Addison's disease 2001. J Clin Endocrinol Metab 86: 2909-2922, 2001.

2 - BETTERLE C; DAL PRA C; MANTERO F \& ZANCHETTA R. Autoimmune adrenal insufficiency and autoimmune polyendocrine syndromes: autoantibodies, autoantigens, and their applicability in diagnosis and disease prediction. Endocrinol Rev 23: 327-364, 2002.
3 - VAIDYA B; PEARCE S \& KENDALL-TAYLOR P. Recent advances in the molecular genetics of congenital and acquired primary adrenocortical failure. Clin Endocrinol 53: 403418, 2000.

4 - LORIAUX DL \& MCDONALD WJ. Adrenal insufficiency. In: DEGROOT LJ \& JAMESON JL, eds. Endocrinology, 4th ed, W.B. Saunders, Philadelphia, p. 1683-1690, 2001.

5 - GRINSPOON SK \& BILLER BM. Laboratory assessment of adrenal insufficiency. J Clin Endocrinol Metab 79: 923931, 1994. 